\title{
Gouvernance et acteurs privés : le cas de la lutte contre les discriminations au travail
}

\section{Romain Huët et Morgane Cantrelle}

\section{OpenEdition}

\section{Journals}

Édition électronique

URL : http://journals.openedition.org/developpementdurable/1880

DOI : 10.4000/developpementdurable.1880

ISSN : 1772-9971

\section{Éditeur}

Association DD\&T

\section{Référence électronique}

Romain Huët et Morgane Cantrelle, « Gouvernance et acteurs privés : le cas de la lutte contre les discriminations au travail », Développement durable et territoires [En ligne], Varia (2004-2010), mis en ligne le 21 juillet 2014, consulté le 19 avril 2019. URL : http://journals.openedition.org/ developpementdurable/1880; DOI : 10.4000/developpementdurable.1880

Ce document a été généré automatiquement le 19 avril 2019.

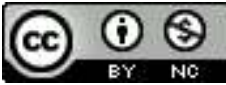

Développement Durable et Territoires est mis à disposition selon les termes de la licence Creative Commons Attribution - Pas d'Utilisation Commerciale 4.0 International. 


\title{
Gouvernance et acteurs privés : le cas de la lutte contre les discriminations au travail
}

\author{
Romain Huët et Morgane Cantrelle
}

1 Le terme de "gouvernance » s'est introduit dans le lexique économique et politique sans qu'on n'y prête attention ou le définisse. Il s'apparente à une expression fourre-tout, comme celui, auquel il est très lié, de « société civile » [Cassen, 2001]. Le terme est d'ores et déjà en place, comme en témoigne la multitude d'ouvrages politiques, économiques ou sociologiques, utilisant ce terme sans jamais le définir. Pourtant, on s'apercevra qu'il ne s'agit pas là d'un simple mot mais d'un élément, non seulement révélateur, mais surtout structurant d'une idéologie. Ainsi, nous appréhendons la gouvernance comme un terme utilisé pour résumer les changements dans les formes d'action publique ou dans les modes de gestion de l'action publique (1) [Muller et Surel, 1998]. Dans un second temps, nous mettrons à l'épreuve ce concept de gouvernance et plus particulièrement certains de ces attributs développés par Guy Hermet [2005] à partir de l'étude de la «Charte de la diversité» dans l'entreprise (2). En définitive, à travers la problématique des « discriminations fondées sur l'origine ethnique » dans le monde du travail, nous montrerons comment une question sociale a été saisie par des acteurs privés, auteurs de différentes initiatives telles que la production de rapports dédiés à ce sujet ( « Les oubliés de l'égalité des chances", "Des entreprises aux couleurs de la France "...) ${ }^{1}$ et à travers la récente signature de la «Charte de la diversité» par une quarantaine d'entreprises françaises. L'analyse de ce cas nous permettra d'esquisser quelques spécificités de ce mode de gestion de l'action publique en mettant en évidence les modalités de fonctionnement de ce collectif et plus largement celles relatives à l'organisation de l'action (3). 


\section{La gouvernance comme l'indice de nouvelles formes d'actions publiques}

2 Notre travail sur la gouvernance consiste à éclaircir ce terme, à identifier certains de ses attributs, à le caractériser, et surtout à le mettre à l'épreuve du terrain (à partir d'une étude de cas sur la «Charte de la diversité» dans les entreprises). Avant de présenter cette étude de cas, il n'est pas inutile de préciser quelques caractéristiques de la gouvernance que nous avons repérées au gré de nos différentes lectures:

3 Il s'agit d'un mode de gestion des affaires publiques ou privées.

4 Son apparition remonte à plusieurs années mais le terme a été sorti de l'oubli en 1989 par les experts des grandes agences internationales de l'aide au développement, Banque Mondiale en particulier, qui prescrivent «la bonne gouvernance» aux dirigeants politiques. Depuis, le terme est régulièrement utilisé par les journalistes ou politiciens.

5 Le terme a trouvé sa place et pris sens sous l'impact de la globalisation en même temps que de la complexité croissante des demandes sociales.

6 La définition que nous retenons dans le cadre de notre travail ${ }^{2}$ est celle de Patrick Le Galès [1998]. Il y voit une idée de conduite, de pilotage, de direction, mais sans le primat accordé à l'État. Il la définit comme un "processus de coordination d'acteurs, de groupes sociaux, d'Institutions pour atteindre des buts propres discutés et définis collectivement dans un environnement fragmenté, incertain ».

7 Mais la diversité des champs d'actions (économique, politique, juridique, financier, environnemental, social, fiscal), et des échelles auxquels le terme s'applique brouille sa compréhension. Ainsi, on peut trouver des ouvrages sur la gouvernance urbaine, la gouvernance de l'ordre juridique international, d'entreprise, d'Internet, de l'écotourisme, de l'administration, du développement local, des transports publics, de la sécurité sociale, de la distribution automobile, sur le lien entre gouvernance et identités, gouvernance et immigration, gouvernance et insécurité, etc. que ce soit au niveau mondial, européen, dans les pays arabes, en Afrique subsaharienne, ou encore au niveau d'une ville. Dans cette diversité, il est difficile d'établir une définition générique. De manière générale, le terme de gouvernance est souvent utilisé pour désigner et résumer l'évolution des formes de l'action publique.

8 Guy Hermet, politologue spécialiste de l'étude de la formation des régimes démocratiques ${ }^{3}$, développe une approche critique de ce concept. Dans son dernier ouvrage [2005, Ibid., pp.8-10], il a déterminé six attributs communs à toutes les applications de la gouvernance, à savoir :

- La gestion des affaires publiques est réalisée comme si leur traitement ne devait pas différer sensiblement de celui des affaires privées. La gouvernance vise à abolir la distinction public/ privé pour ne plus réserver à l'État, et ses organisations que le rôle d'agents régulateurs (plutôt que d'autorités régulatrices).

- Ce rôle de simple agent régulateur est justifié par la croyance que, dans tous les domaines, les sociétés ou les relations entre pays sont régies par des mécanismes d'auto-ajustement apparentés à ceux du marché sur le plan économique. L'agent régulateur doit juste réglementer la concurrence et confondre les tricheurs. L'objectif des adeptes les plus radicaux de la gouvernance (que l'on retrouve généralement parmi les économistes 
libéraux) est de soustraire les décisions dites "sérieuses ", c'est-à-dire économiques, à la politique et aux politiciens réputés ignorants, démagogues et irresponsables.

- Les acteurs décisifs des dispositifs de gouvernance se recrutent ou se choisissent entre eux, par cooptation avant tout en fonction de leurs positions acquises ou de celles qu'ils parviennent à acquérir grâce à leur talent tactique et à leur proximité, idéologique, avec les acteurs déjà en place. S'ils le font encore, la procédure de l'élection et le principe de la représentativité élective n'interviennent plus que de façon résiduelle.

- La gouvernance correspond à un processus de décision toujours révocable et provisoire, elle ne désigne pas le site du pouvoir ultime et exclusif des autres comme le font les notions de gouvernement ou d'Etat.

- Les décisions ne sont plus le produit d'un débat et d'une délibération. Elles sont le résultat de négociations, voire de marchandages et de trocs entre les différentes parties.

- La gouvernance est un mode de gestion qui tend à se codifier au regard de normes ou de « codes de conduite » négociés plutôt que de lois votées en vertu du principe majoritaire, ou issues d'une tradition jurisprudentielle illustrée par la Common Law.

C'est à partir de ce dernier attribut, posé comme postulat de départ, que nous avons choisi de nous intéresser à un document de type charte/code. Les chartes constituent un objet communicationnel relativement ambivalent et dont l'extrême hétérogénéité des formes et des contenus peut parfois paraître déroutante. Notre travail de Master Recherche puis de Doctorat montre qu'une approche sémio-pragmatique de ces documents n'est pas suffisante pour clarifier leur hétérogénéité [Huët, 2005]. En réalité, il n'existe aucune unité formelle entre ces documents, aucun «statut pragmatique évident ", mais une pluralité d'objets qui renvoient à des situations sociopolitiques fondamentalement différentes. La production d'une charte dans l'entreprise (code éthique, charte de valeurs produites unilatéralement par le sommet hiérarchique), par un collectif d'entrepreneurs (c'est le cas de la "Charte de la diversité»), sous l'égide d'un régulateur international (Global Compact, principes directeurs de l'OCDE, etc.) ou directement par les parties prenantes (nombre de chartes sont écrites directement par les parties prenantes et appellent de manière organisée l'entreprise à assumer ses responsabilités sur certaines thématiques sociales ou environnementales) impliquent et génèrent des rapports sociaux différents, des lieux et des partenaires de négociation variés, des efficacités également inégales, etc. Ainsi, l'analyse du processus de production est proposée comme étant une "clé de lecture » pour saisir ce que signifie l'objet charte [Huët, 2006].

Pour Guy Hermet, les chartes et codes sont intimement liés à la notion de gouvernance, ils la « codifient ». En octobre 2004, nous avons pu lire dans la presse française que 35 grandes entreprises avaient signé la «Charte de la diversité» de l'Institut Montaigne. A partir de l'analyse du processus de production de cette charte, nous mettons à l'épreuve du terrain les attributs de la gouvernance afin de montrer que l'apparition de la charte dans l'espace public constitue en soit un acte de gouvernance. Nous verrons notamment qu'étudier la gouvernance exige de comprendre l'articulation des différents types de régulation sur un territoire, et de réexaminer les interrelations entre "société civile ", État, marché, et les recompositions entre ces sphères dont les frontières se brouillent. 


\section{La « charte de la diversité » comme mode de gestion de la question des discriminations}

\subsection{Présentation de la «Charte de la diversité »}

1135 dirigeants de grandes entreprises ont signé le 22 Octobre 2004 une charte qui s'intitule «charte de la diversité dans les entreprises ». Les entreprises s'engagent à «Rechercher une diversité au travers des recrutements et de la gestion des carrières». Il s'agit de mettre en place des mesures volontaristes pour que l'entreprise reflète les composantes de la société, notamment en recrutant plus largement les personnes issues de l'immigration ou des Départements d'Outre Mer (DOM). Cette charte cherche donc à pallier l'incapacité des pouvoirs publics à faire face au défi de l'intégration des populations immigrées.

La «Charte de la diversité» est produite à l'initiative de l'Institut Montaigne ${ }^{4}$ et plus précisément sous l'égide d'un groupe de travail composé de deux acteurs: Laurence Méhaignerie et Yazid Sabeg. Elle est une des propositions faites dans le rapport Les oubliés de l'égalité des chances [2004] par ces mêmes acteurs. Le groupe de travail a élaboré une première version de charte qui a ensuite été discutée avec quelques dirigeants de grandes entreprises au sein de l'AFEP ${ }^{5}$ (Association Française des Entreprises Privées) qui constitue le lieu de négociation. Cette charte a donc été discutée directement avec une vingtaine de chefs d'entreprise, qui ont apporté des commentaires. La charte a ainsi évolué au cours de quatre rencontres, avant d'être stabilisée, signée, puis rendue publique.

\subsection{La charte comme support d'une communication « auto- référentielle »}

13 Ce document renvoie indirectement à la manière dont les entreprises participent, selon différents niveaux territoriaux, à un développement respectueux de l'environnement social. Sous l'effet de multiples facteurs que nous ne pouvons détailler ici (voir Capron et Quairel Lanoizelée, Descolonges et Saincy, De la Broise et Lamarche, etc. $)^{6}$, les entreprises sont amenées à se justifier davantage sur la manière dont elles entendent s'intégrer concrètement dans la société. Dans cette perspective, l'article 116 de la loi Française sur les Nouvelles régulations économiques (2001) a impulsé parmi les entreprises admises aux négociations sur un marché réglementé un important mouvement de formalisation des impacts sociaux et environnementaux. Parmi cette panoplie discursive, les chartes constituent un support pour diffuser un discours sur la manière dont l'entreprise entend s'intégrer dans la société.

14 Ainsi, cette étude de cas sur la «Charte de la diversité» peut aisément s'inscrire dans une problématique plus générale qui a trait à la Responsabilité sociale des entreprises. Dans les perspectives ouvertes par D'Almeida [1999, 2001] Le lien entre la RSE et sa communication peut se comprendre à travers l'analyse de l'intégration -par les acteurs économiques- de discours reposant sur des valeurs élargies qui dépassent la simple « communication produit ». En réalité, les discours de l'entreprise sur sa responsabilité relèvent conjointement des valeurs internes locales qui sont mises en adéquation avec des valeurs externes, voire dans certains cas universelles. Comme le montre l'auteur [ibid.], les acteurs économiques en sont venus à produire un discours universel en 
élargissant leur cadre d'action et en empruntant notamment aux valeurs fondatrices des institutions d'intérêt général $l^{7}$. A travers cette «Charte de la diversité», l'entreprise affiche un souci quant aux préoccupations sociales. C'est ce qu'exprime la notion "diversité " (titre de la charte) qui désigne en réalité sur le plan communicationnel un discours " auto-référentiel ». Nous verrons que celui-ci tend à s'appuyer sur des valeurs d'intérêt général et des missions de service public. On a donc un discours qui prétend référer aux principes de cohésion et de justice sociales qui seraient alors assurés par les entreprises.

Or, ce que nous disons tend à accréditer l'idée selon laquelle les chartes servent de support de cette communication « auto-référentielle » en engageant une conception du devoir que se donnent les entreprises. La charte revêt un engagement institutionnel qui structure sa communication sur un registre qui fait appel au système de la promesse. En définitive, une charte constitue une promesse de l'entreprise faite à ses parties prenantes. A travers cette volonté de «s'auto-prescrire » des règles (en dehors de l'autorité légale), nous pouvons remarquer que la «Charte de la diversité» formalise non seulement un engagement, mais constitue aussi l'expression d'un « devoir prescrit à soi » [D'Almeida, 2001, ibid.). Néanmoins, notre travail consiste à montrer que la charte ne constitue pas seulement un " jeu de langage», mais engage au contraire une conception militante du devoir que formulent les énonciateurs.

\subsection{Analyse juridique des chartes}

Sur le plan juridique, l'objet « charte » est à de rares exceptions près normatif. À l'origine, les chartes se sont développées aux États-Unis. Les gouvernements successifs ont incité les entreprises à développer des chartes formelles. La publication des "Federal Sentences Guidelines" (1991) constitue un texte de loi qui prévoit de réduire les peines des sociétés jugées pénalement responsables si les entreprises disposent d'un code de conduite et si elles font la preuve de leurs actions pour assurer le respect des programmes de conformité aux normes (obligation de moyen pour obtenir une réduction de la sanction). Ainsi, comme le notent Ballet et De Bry [2001] le modèle américain des chartes est un modèle subordonné à l'existence de dispositifs juridiques. Ces documents intègrent l'ensemble ou une partie des réglementations existantes sur un thème donné. C'est donc une logique qui vise à mettre en conformité les comportements et les règles de l'entreprise avec le corpus juridique en vigueur. Ceci explique en partie le développement des « chartes américaines » qui sont utilisées par les entreprises car elles sont prises en compte dans la fixation du quantum de la sanction. En revanche, pour le droit français, cette justification juridique n'a pas de sens car la loi ne reconnaît pas ce type de document [Ballet, De Bry, ibid.]. Cependant, selon Isabelle Desbarats [2003], une charte peut disposer d'un régime juridique connu. Dans le droit français, elle peut être assimilable à un règlement intérieur et peut ainsi avoir force obligatoire sous réserve que soient respectées les conditions tenant à son contenu et à son élaboration. Elle peut également avoir une portée contractuelle si ses dispositions sont intégrées au contrat de travail, soit lors de la conclusion de celui-ci, soit par le biais d'un avenant ultérieur. À l'inverse, si ces documents peuvent accroître des obligations pesant sur les salariés, ils peuvent également comporter des nouvelles prérogatives au personnel, constituant autant de nouvelles obligations à la charge de l'entreprise. Les juges peuvent «détecter, dans ces documents, de nouveaux droits au profit des salariés plutôt que de nouvelles obligations à leur charge. D'abord, ce sont les standards classiques du droit (faute, fraude, dol, bonne foi) qui pourraient conduire le juge à conférer des effets juridiques aux engagements pris dans ce type de 
codes. D'autre part, rien n'interdit de penser que les principes énoncés dans un code, comme les engagements susceptibles d'y être pris par l'employeur au profit de son personnel, peuvent acquérir une force juridique grâce à la qualification d'usage d'entreprise. C'est, enfin, le mécanisme de l'engagement unilatéral de volonté de l'employeur qui est susceptible de l'engager à l'égard des salariés» [Desbarats, 2003, pp.338-339]. Cependant la question de la valeur juridique est actuellement peu pertinente dans la mesure où ces documents sont généralement élaborés en marge des sources reconnues du droit (origine unilatérale) et d'autre part, leurs contenus étant relativement généraux, le contrôle du respect des engagements (souvent non prévu) est difficilement applicable. C'est typiquement le cas de la «Charte de la diversitén ${ }^{8}$.

17 Ainsi, l'ambiguïté du document réside dans les tensions qui existent entre la force politique $^{9}$ du texte (provenant de la signature de grandes entreprises internationales, et de sa médiatisation) et le fait qu'elle ne dispose d'aucun caractère juridique contraignant ${ }^{10}$. L'absence d'inscription des chartes dans le langage du droit n'est pas sans susciter des interrogations, car comme le note Claude Lefort [1981], « À partir du moment où les droits de l'homme sont posés comme ultime référence, le droit établi est voué au questionnement. Il fait toujours question, à mesure que les volontés collectives, ou si l'on préfere, que des agents sociaux, porteurs de revendications nouvelles mobilisent une force en opposition à celle qui tend à convertir les effets des droits reconnus (...) Tocqueville montrait également qu'à peine close, une lutte politique en ouvre une autre; les droits conquis par un groupe social offrent l'exemple d'une revendication aboutie qui encourage la mobilisation d'autres groupes. En d'autres termes, le fait de poser explicitement par écrits des droits, suscite un certain engouement dans l'attention portée à une question donnée, c'est-à-dire que chacun des partenaires concernés est susceptible de mettre en exergue les lacunes du texte. Ainsi, une charte qui ne se contente pas d'énoncer mais qui implique dans "une dynamique de droits» est plus puissante car elle fera l'objet de véritables discussions par les groupes sociaux » [Lefort, 1981, pp.67-68].

\subsection{La «Charte de la diversité» ne se substitue pas à la loi : retour sur le corpus juridique en vigueur}

Pour revenir au cas spécifique de la «Charte de la diversité», il est essentiel de noter qu'elle apparait dans un arsenal juridique principalement issu du droit communautaire, telle que la directive 2000/43/CE relative à l'égalité de traitement sans distinction de "race » ou d'origine ethnique, et la directive 2000/78/CE qui concerne plus spécifiquement l'égalité de traitement en matière d'emploi et de travail. Leur application en France dans la loi du 16 novembre 2001 a considérablement renforcé le cadre juridique existant, modifié le code du travail et crée la Haute Autorité de Lutte contre les Discriminations et pour l'Egalité (HALDE). Cette autorité administrative indépendante peut être saisie directement pour des faits de discrimination, elle promeut la recherche, encourage les démarches volontaires en matière d'égalité des chances, et donne des avis et recommandations sur d'éventuelles modifications législatives et réglementaires. Ainsi, la «Charte de la diversité» n'émerge pas dans un cadre « d'anarchie normative » ou de désert « juridique ». Par ailleurs, ce document ne se substitue pas à la loi. Il participe en revanche à repousser les intentions gouvernementales. La charte est l'occasion de plaider pour une phase d'expérimentation qui donne la priorité au volontariat et à la responsabilité de l'entreprise. Elle s'apparente à du militantisme entrepreunarial dans la mesure où sa signature permet aux entreprises de se prémunir contre toute accusation de pratiques 
discriminatoires et témoigne de leur engagement public. Dans un même temps, il s'agit d'une forme de pression de ces acteurs qui se constituent en lobby ${ }^{11}$ pour anticiper ou faire évoluer la législation dans ce domaine.

On retrouve cette tentative du pouvoir économique d'influer sur les régulations étatiques chez Catherine Loneux [2000]. À travers une étude sur l'interprofession publicitaire, elle montre comment l'entreprise à travers ses discours éthiques impose ses propres critères de fonctionnement, notamment en matière juridique, et comment ceux-ci sont ensuite appropriés et respectés par les entreprises, via l'auto- proclamation du BVP, Bureau de Vérification des Publicités. Selon les entreprises, grâce aux discours éthiques auto promus; « La publicité a gagné en terme d'image, elle s'est créée elle-même une place de choix dans la société. L'interprofession publicitaire a su se structurer fortement à partir de significations sémantiques communes et autour de l'autorégulation, principe promu entre autres par la chambre de commerce international (CCI) qui sert d'organisme centralisateur aux professionnels des affaires. Les instances représentatives de la profession publicitaire ont construit une réponse à ce qu'elles appréhendent comme une menace réglementaire, en mettant en place une stratégie de discours éthiques doublée d'actions de lobbying" (Loneux, 2000). Le cas présenté par Catherine Loneux est relativement similaire à cette "Charte de la diversité». Il s'agit à la fois d'objectiver une problématique sociale qui doit, selon les milieux patronaux, figurer dans l'agenda politique en utilisant l'alibi de la bonne foi, du volontarisme, de la légitimité de ce groupe professionnel à s'autosaisir d'une question sociale et à s'autoréguler en cherchant à terme, à anticiper ou à prévenir les intentions gouvernementales. Sur cette question des discriminations, l'Institut Montaigne, et plus largement le monde des entreprises, se sont positionnés comme des interlocuteurs incontournables pour les instances de réglementation.

20 Enfin, l'apparition de la charte a d'ores et déjà apporté des changements dans le paysage de la lutte contre les discriminations. Ce n'est pourtant pas la première fois qu'une telle démarche est entreprise, avec des différences de contenu néanmoins, mais force est de constater que les quelques chartes de lutte contre les discriminations portées par les pouvoirs publics n'ont pas eu le succès de cette dernière. Outre l'impact médiatique lui conférant un climat de diffusion propice, elle a surtout l'avantage d'être initiée par des chefs d'entreprise, ce qui la rend légitime aux yeux des destinataires qui perçoivent moins l'objet comme une contrainte. L'Institut Montaigne a donc réussi là où les politiques avaient échoué. Encore aujourd'hui, cette question reste difficile à traiter politiquement. La charte a donc permis à ce que le sujet sorte de son carcan politique pour laisser place à un débat sociétal (ex: sur le CV anonyme, autre proposition de l'Institut Montaigne, ou sur d'autres initiatives en cours telles que le label de la diversité, la possibilité de réaliser une photo statistique de la composition sociologique de l'entreprise, etc.), les politiques se contentant d'approuver, d'inciter, et de laisser faire cette «dépolitisation» de la question. La loi Borloo reconnaît la «Charte de la diversité» de l'Institut Montaigne, et lui laisse deux ans pour faire ses preuves, et prévoit un débat au Parlement à ce sujet s'il n'y a pas eu d'avancées durant ces deux années. Ainsi, l'Institut Montaigne et son réseau d'entreprises sont devenus des acteurs pertinents reconnus, ou au moins reconnus comme existant dans la définition des politiques publiques sur cette question des discriminations.

21 Il s'agit désormais pour nous de vérifier, par cette étude de cas, nos hypothèses de départ. Nous montrerons tout d'abord que la «Charte de la diversité» s'inscrit dans une idéologie 
libérale, en illustrant les propos de Hermet selon lesquels «La gouvernance vise à abolir la distinction public/privé pour ne plus réserver à l'État, et ses organisations que le rôle d'agents régulateurs. (...) Ce rôle est justifié par la croyance que, dans tous les domaines (...) les relations (...) sont régies par des mécanismes d'auto- ajustement apparentés à ceux du marché sur le plan économique».

\section{Idéologie sous-jacente et rôle de l'État.}

Pour ce faire, nous avons étudié le contexte d'apparition de la charte et la façon dont les acteurs ont objectivé la problématique des discriminations.

\subsection{Un sujet politique porté par des acteurs privés}

C'est tout d'abord au regard d'une participation croissante des acteurs privés dans les décisions politiques que l'abolition de la distinction public/privé, qui justifiait le rôle des pouvoirs publics au nom de l'intérêt général, se réalise progressivement. Ces acteurs privés estiment être les mieux placés pour prendre des décisions ayant une influence sur «leur» milieu, et prétendent qu'il est possible de prendre des décisions économiques sans faire de politique.

La dépolitisation de la question des discriminations, que cristallise cette charte, s'explique aussi par un certain désengagement de l'État sur cette question. À titre d'exemple, nous avons observé dans les plans départementaux de lutte contre les discriminations en 2005, que le département du Nord affiche une priorité à la lutte contre le racisme alors que ce type de discrimination - directe - est largement minoritaire face à la discrimination indirecte et systémique qui nécessite une réelle remise en cause du fonctionnement de la société. De la même façon, les principales mesures de lutte contre les discriminations sont destinées aux conditions d'accueil et d'insertion des personnes étrangères, preuve qu'il est plus facile de mettre en place et valoriser des actions en faveur des étrangers, plutôt que d'avouer que la France discrimine une partie de ses Français. L'État affirme quant à lui son soutien aux initiatives associatives et se contente d'annoncer le lancement d'une "grande campagne de communication destinée à sensibiliser à la diversité dans les entreprises en période de pénurie de main d'œuvre ». Un tel contexte entraîne la suspicion et favorise les actions associatives et militantes. C'est également dans ce contexte que la question des discriminations fut mise au goût du jour sur un ton dénonciateur par le milieu entrepreunarial en la personne de Yasid Sabeg, qui créa la surprise aux Assises de la politique de la ville ${ }^{12}$.

\subsection{Objectivation de la problématique des discriminations}

Pour «faire entendre» le propos de la charte, la diffusion d'un système de valeurs et la construction d'une certaine réalité étaient préalablement nécessaires. C'est dans cette optique que deux rapports sur ce sujet ont été rédigés par l'Institut Montaigne, dont un commandité par le Premier Ministre. La «Charte de la diversité» n'objective donc pas à elle seule une problématique dans l'espace public, mais elle y participe et prétend apporter des solutions à ce problème. ont été rédigés par Claude Bébéar (pour le premier), et Laurence Méhaignerie et Yazid 
Sabeg (pour le second). Ces derniers seront également les rédacteurs de la charte. Le premier rapport cherche à objectiver la charte en tant que « solution» alors que le second tend à confirmer sa pertinence.

Ces écrits dressent un état des lieux orienté ${ }^{13}$ des discriminations en France et dans les entreprises. Ils formulent une série de propositions pour faire évoluer le comportement des entreprises et du monde politique sur cette question. Parmi le panel de suggestions, l'une propose l'élaboration d'une charte qui serait signée par une grande partie des entreprises françaises ${ }^{14}$. Par ailleurs, dans le second rapport, les auteurs prennent position en faveur de la discrimination positive qui «n'a rien à voir avec la discrimination à rebours. A l'égalité formelle, elle préfere l'égalité réelle. En ce sens, elle est non seulement conforme au principe de justice, mais elle est également un moyen de l'instaurer » [Sabeg, Méhaignerie, Ibid.] Ils utilisent également le terme (inspiré du modèle canadien) de "minorités visibles " pour cibler les personnes qui font l'objet de discriminations. Il s'agit pour les acteurs de mettre en exergue, de façon engagée, la spécificité de leurs approches de la question des discriminations.

Selon le sociologue Philippe Bataille, spécialiste des questions d'intégration et de lutte contre les discriminations, ces rapports sont «Le début de la prise de conscience de ce problème des discriminations à l'échelle de l'entreprise, mais ça ne va pas beaucoup plus loin » ( $\mathrm{La}$ Tribune du 26/11/2004). En réalité, nous pouvons nous apercevoir que ces deux rapports marquent une évolution significative dans le débat public sur ce domaine en lui conférant un certain ordre dans l'explication des phénomènes.

Or ces explications particulières du réel tendent à justifier les actions préconisées dans les rapports, et le rôle particulier joué par les entreprises. Les auteurs montrent ainsi que la charte fait partie d'un dispositif plus large qui vise à prouver la capacité des entreprises à s'autosaisir d'une question sociale et à s'autoréguler. Mais pour ce faire, l'Institut Montaigne insiste sur la nécessité d'une approche «pro-active » des entreprises. Ainsi, la charte sous-tend une conception précise de la régulation et du rôle de l'Etat. En ce sens elle peut être un révélateur des transformations structurelles de l'action publique et de l'état des rapports de force entre les partenaires sociaux. Comme nous avons pu le voir, la charte ne se substitue pas aux réglementations en vigueur. Pour autant, les signataires appellent à s'engager dans des « actions positives à la française » sur une base volontaire. On est donc dans une régulation incitative et non normative. Par ailleurs, l'émergence de la charte s'accompagne d'un discours de "délégitimation» des instruments de l'action publique. Ceux-ci sont considérées comme inefficaces et peu adaptées au contexte de l'entreprise (incapacité à assurer le respect de la réglementation, à promouvoir la diversité dans les entreprises, y compris en son sein avec des critiques récurrentes sur la gestion des discriminations au sein des administrations publiques, etc.). A titre d'exemple la loi sur le CNIL est fortement contestée car elle ne permet pas aux entreprises d'établir une photographie statistique et d'évaluer les progrès accomplis en la matière ${ }^{15}$. Dans cette même perspective, d'autres normes sont en cours d'élaboration. L'une d'entre elles consiste en la mise en place d'un label de la diversité dont l'obtention par les entreprises pourrait à terme déboucher sur des réductions fiscales. En tout état de cause, ces différentes initiatives participent à une forme de dépolitisation du problème au profit du bon fonctionnement du collectif et du pragmatisme. Le rôle « d'agent régulateur » est délégué à d'autres acteurs (les médias, les consommateurs, etc.) voire à d'autres organismes qui sont en train de créer et gérer le «marché de la diversité » (Institut du Mécénat et de la Solidarité, Entreprise et Personnel, etc.). 
30 Ainsi, l'Institut Montaigne joue ici son rôle de groupe de pression, en cherchant à assouplir certaines dispositions qui contraignent l'entreprise et qui nuisent à son action pour lutter contre les discriminations. Dans le rapport de Claude Bébéar [2004, ibid.], figurent un ensemble de propositions intitulées « Des initiatives que l'entreprise peut prendre seule ». C'est dans cet ensemble de propositions que figure l'annonce du CV anonyme qui a fait l'objet d'une très large médiatisation. Cette partie est également l'occasion aussi de mettre en avant quelques initiatives "exemplaires» d'entreprises "qui méritent d'être multipliées à grande échelle». C'est finalement sous l'effet de ce lobbying entrepreunarial que le sujet des discriminations au travail sera abordé dans la loi de Cohésion sociale de Borloo, qui reconnait la charte et accorde une période d'expérimentation de deux ans aux entreprises. La conférence gouvernementale sur l'égalité des chances organisée par Matignon (3 Février 2005) va également dans ce sens. L'État s'y est notamment engagé à :

- soutenir les initiatives des partenaires sociaux et des entreprises en matière de promotion de l'égalité, dans le domaine de la lutte contre les discriminations à l'emploi et la formation

- se mobiliser en son sein en tant qu'employeur

- lancer une grande campagne de communication sur les enjeux de la diversité en période de pénurie de main-d'œuvre.

31 En définitive, l'État ne joue qu'un rôle de soutien et d'incitateur. Il invite notamment les partenaires sociaux à négocier sans pour autant fixer le cadre ce ces négociations et déclare, au cours de cette conférence : "Il appartient aux partenaires sociaux de déterminer les niveaux de négociation les plus appropriés pour promouvoir l'égalité des chances (...) La relance de la négociation collective à ce niveau n'exclut pas la recherche d'un accord national interprofessionnel sur ce thème, ni bien entendu, d'accords au niveau des entreprises" [Conférence gouvernementale sur l'égalité des chances, 3/02/2005].

Le gouvernement accorde donc explicitement le primat à l'autorégulation des entreprises sur cette thématique et fixe une échéance où cette phase d'expérimentation sera évaluée. Il en découle, pour lui, un simple rôle d'agent régulateur, comme nous le supposions au commencement de notre travail.

33 Ce rôle est bien justifié par la croyance dans les mécanismes d'auto-ajustement, dans les affaires publiques, comme pour le marché, ce qui est caractéristique de l'idéologie libérale. La charte et les rapports laissent à penser en effet qu'en s'attaquant à cette question sous l'angle de problèmes économiques, les solutions apportées au profit d'acteurs particuliers aura des conséquences positives au niveau politique et social, pour l'égalité des chances et donc dans l'intérêt général. En pensant que si les entreprises embauchent des personnes habituellement défavorisées, la question de l'intégration sera résolue et la discrimination disparaîtra des mentalités, cette action s'inscrit pleinement dans les théories de l'insertion par l'économique ${ }^{16}$.

Entre autres caractéristiques du libéralisme figurent le culte du pragmatisme et de la méritocratie. Ainsi pour Michel David, Président d'un centre de ressources sur l'intégration et la lutte contre les discriminations, la charte et les rapports s'inscrivent "dans la lignée d'une philosophie libérale accordant le primat à l'intégration économique, à l'initiative, au mérite par la constitution d'élites reconnues issues des "minorités visibles", (...) Or, la seule promotion des diplômés et donc des classes moyennes issues de l'immigration, ne suffit pas à réaliser l'égalité des chances. $\rrbracket^{17}$.

L'exemple de cette charte illustre bien comment la situation éminemment politique des personnes discriminées en France a été érigée en problématique économique. La 
signature de la charte par un nombre important d'entreprises est également une manière de témoigner de la capacité des entreprises à s'autoréguler. En définitive, la communication joue un rôle clé dans le processus. En effet, la charte constitue en soi un cadre de production collective homogène qui oriente la nature, les objectifs et les attendus des débats. La communication est centrale dans la mesure où ce qui importe, c'est l'action de "dire ensemble » et moins «l'objet du dire » c'est-à-dire ce que font réellement les entreprises. Il s'agit donc d'une phase d'objectivation d'un fait social et des solutions qui s'y rapportent. En outre, selon Claude Bébéar, «L'expérience est probante car les (...) entreprises partenaires ont perçu tout l'intérêt qu'il y avait à prendre en considération les candidatures émanant des minorités visibles ». Ainsi, nous pouvons nous apercevoir que cette action contribue à labelliser le problème dans un sens particulier, révélateur d'un système de valeurs accordant au pragmatisme et aux acteurs économiques une place cruciale dans l'action publique.

\subsection{Précarité des décisions et logiques de cooptation}

Ces premières hypothèses vérifiées, nous nous intéressons désormais aux processus décisionnels pour voir s'ils sont, comme le prétend Guy Hermet, révocables, provisoires, résultant de négociations plus que de débats démocratiques. Pour étudier les points évoqués, la reconstitution rigoureuse du processus d'élaboration de la charte par étapes a été nécessaire. La stratégie de diffusion sera quant à elle plus révélatrice des logiques de cooptation.

\subsubsection{Le processus d'élaboration de la charte : négociation et compromis au sein d'un collectif de travail}

Nous n'avons pu assister à l'élaboration de la charte puisqu'elle est antérieure à notre recherche et a été réalisée dans le lieu très clos qu'est l'Association Française des Entreprises Privées (AFEP). Mais nous nous sommes entretenus avec la rédactrice de la charte et nous avons récolté différentes "traces discursives » issues des rencontres entre les acteurs. A titre d'exemple, nous disposons de l'ensemble des versions de charte (des différentes versions intermédiaires), les lettres qui accompagnaient la diffusion de la charte, les argumentaires, les divers documents de travail, etc.

Il s'avère que 3 versions différentes de la charte ont été rédigées au sein de l'Institut Montaigne. La troisième a été présentée au collectif d'entrepreneurs membres de l'A.F.E.P le 26 mai 2004 accompagnée d'un courrier explicatif (ce document a été envoyé à l'ensemble des membres de l'association). Il est signé conjointement par Yasid Sabeg et Claude Bébéar. Il sert de justification sur l'intérêt de l'implication des entreprises sur ce projet de charte. Les auteurs expliquent ; "Un plafond de verre bloque l'ascension sociale de nombre de nos concitoyens, discriminés à raison de leur patronyme, de leur couleur de peau ou de leur appartenance supposée à un groupe ethnique. Ils sont exclus de pans entiers de la sphère économique et de la vie publique. Nous discriminons le plus souvent sans le savoir (...) cette diversité doit être visible, mais est aussi d'intérêt national ».

En opérant un détour par la théorie des conventions de Boltanski et Thévenot (1991), cette lettre d'accompagnement et la charte sont des documents qui stipulent ce qui est considéré comme juste. La charte définit les conditions pour qu'une pratique soit jugée acceptable, et la lettre d'accompagnement sert de justification à ce sens du juste. La 
formulation selon laquelle la diversité est une question "d'intérêt national » (Lettre d'accompagnement de la charte), et est symptomatique de la prégnance d'une argumentation relevant du monde civique. Il s'agit bien d'un renoncement à l'intérêt particulier. Dans un même temps, les arguments qui suivent mettent en avant une justification fondée sur l'efficacité économique: «La «Charte de la diversité» dans l'entreprise repose sur une idée simple. Nos entreprises ont intérêt à refléter la diversité de la société dans laquelle elles évoluent. Favoriser la diversité dans l'entreprise est une démarche volontariste qui demande un engagement à chaque échelon de l'entreprise. De la même façon que la mixité hommes femmes au travail, est de fait, un facteur de dynamisme social et un stimulateur de performances, la diversité relève non pas de la compassion mais bien de l'intérêt économique et social de l'entreprise (...) dans d'autres pays, les entreprises qui ont placé la gestion de la diversité au cœur de leur stratégie ne souhaitent pas faire marche arrière. Elles sont conscientes que la diversité dans nos sociétés est devenue une force, un avantage, tant sur le plan économique que social, dont l'impact se fait sentir sur la créativité et la compétitivité des entreprises » (Lettre d'accompagnement de la charte).

40 À travers ces deux justifications, nous pouvons identifier une tension entre «l'exigence de commune humanité » (nécessité d'une reconnaissance d'une égalité fondamentale entre les êtres humains) et cette logique de la performance (la diversité est appréhendée comme un facteur clé de succès). En ce sens, la charte est le résultat d'un compromis. Celui-ci s'inscrit dans l'objet pour éviter que l'on pose à tout instant sa cohérence logique. L'articulation de ces deux niveaux de justification est nécessaire pour que l'objet soit signé et valorisé auprès d'un public plus large (cette lettre sera d'ailleurs jointe au dossier de presse qui a été envoyé quelques jours avant la signature officielle de la charte).

41 Les quatrième, cinquième et sixième versions ont toutes été soumises à la ratification des membres de l'AFEP, ce qui signifie que leur contenu et les modifications opérées sont le résultat de la négociation entre les entreprises. Par conséquent, nous repérons quatre temps de régulation. Nous n'allons pas ici énumérer toutes les modifications, suppressions et ajouts qui ont été réalisés. À titre d'exemple, une question aussi importante que celle de la nature des discriminations traitées (seulement raciales ou ouverte à d'autres «handicaps») a été discutée plusieurs fois. Un des rédacteurs de la charte nous a confié qu'il était parfois difficile d'avancer, les entreprises étant un jour prêtes à de profondes remises en cause, la fois suivante elles reculaient de trois pas. Ainsi, à l'issue d'une réunion, il est arrivé que le contenu se borne à répéter le texte de loi, alors qu'à la séance précédente, les engagements dépassaient ce cadre. Il a même été question d'inciter à des pratiques de discrimination positive illégales en France. Même la question des moments de la carrière d'un salarié où la non-discrimination doit être appliquée a été discutée. Les entreprises pouvaient alors s'engager à remettre en cause les pratiques de recrutement, mais pas leur politique de promotion par exemple. Enfin, l'introduction rappelant un contexte et des principes a souvent été modifiée pour finalement être supprimée.

Le contenu du document a donc été largement discuté, négocié en plusieurs temps et dans des endroits différents. Les différentes versions sont autant de décisions révocables selon le bon vouloir des négociants et de compromis précaires. La charte elle-même relève d'une dimension expérimentale et donc révocable. Mais elle est aussi le fruit de l'homogénéité d'un groupe social de référence, les chefs d'entreprise (même si l'écriture du document est à l'initiative d'un groupe du travail, qui reste néanmoins très proche du milieu patronal $)^{18}$. Elle a été élaborée et décidée dans un cercle restreint et clos selon un 
mode de production aléatoire où les intérêts particuliers d'un collectif sont susceptibles de prendre le dessus sur le principe de bien commun. Les organisations syndicales des travailleurs et les structures associatives sont exclues de la démarche. Elles interviendront plus tard dans la stratégie de diffusion de la charte, dont nous allons rendre compte ultérieurement.

Outre ces échanges opérés au cours de l'élaboration de la charte, des mouvements relationnels se sont créés lors de la signature et la diffusion de la charte. Nous verrons que même après le processus décisionnel, les logiques de cooptation perdurent puisque les acteurs se connaissent et tendent à se choisir.

\subsubsection{La stratégie de diffusion de la charte}

44 L'élaboration de la charte a donné naissance à ce que l'on peut appeler un réseau de la diversité, composé dans un premier temps de rédacteurs de différents rapports sur les discriminations au sein de l'Institut Montaigne, avant de s'ouvrir aux membres de l'A.F.E.P. Lors de la première session de signatures, le réseau s'élargit encore et un questionnaire est envoyé à tous les signataires: ce réseau permettrait en effet aux entreprises d'échanger sur leurs pratiques respectives. Le questionnaire permet de recenser les «bonnes pratiques » mises en place par les entreprises. L'exemplarité est ici recherchée.

En réalité, cette signature officielle du 22 Octobre 2004 va être réitérée dans différentes régions de France. En effet, l'Institut Montaigne, ou des acteurs associatifs, sollicitent les délégations régionales des entreprises initialement signataires pour que celles-ci signent une nouvelle fois cette charte. Dans certains cas, de nouvelles entreprises se rallient au processus. Il s'agit en quelque sorte d'une cérémonie d'un genre particulier, où journalistes, universitaires, responsables associatifs, chefs d'entreprises, et syndicalistes sont invités dans un lieu à caractère plus ou moins prestigieux. Nous avons assisté à l'une de ces « cérémonies » (celle de Lille, organisée au « Grand Palais » le 29 Janvier 2005).

Désormais, des acteurs autres que l'entreprise participeront à sa diffusion. Des associations spécialisées dans la lutte contre les discriminations se rallient à l'initiative des entrepreneurs, en évoquant néanmoins des schémas explicatifs et des intentions qui peuvent entrer en conflit avec celles qui étaient au fondement de la charte. La diffusion de la charte marque son appropriation par des acteurs locaux variés, voire la naissance d'une sorte de "marché de la diversité", composé notamment d'autres associations présidées par Bébéar. L'une, l'Institut du Mécénat et de la Solidarité, est présentée aux signataires comme étant la seule à réaliser des audits de la diversité, étape nécessaire avant de mettre en place quelconque action. L'autre, Des Entreprises aux couleurs de la France, étant l'organe annoncé pour élargir le réseau et surtout suivre et coordonner les actions locales. D'autres associations partenaires agissent dans d'autres régions pour sensibiliser à la diversité et diffuser la charte. A ce titre, l'association Alliances ${ }^{19}$ a réalisé une brochure de sensibilisation intitulée " Prenons goût à la diversité ». Des partenariats se nouent, ou tentent de se nouer. Ainsi Alliances a contacté plusieurs organisations syndicales régionales pour les associer à son projet de diffusion de la charte. Dans la région Nord-Pas-de-Calais, seule Force Ouvrière a accepté de joindre son logo à cette charte.

Enfin, nous avons assisté à l'émergence du CREAP, Centre Régional d'Action Positive, au cours de la cérémonie de Lille. Son auto- proclamation en «centre régional» n'est pas sans 
rappeler celle du BVP, Bureau de Vérification des Publicités que nous avons évoqué précédemment en citant Catherine Loneux. Cette association se positionne également sur ce «marché de la diversité ", en compétition avec l'I.M.S et Alliances. Mais surtout, en prenant le nom de centre régional, elle prétend se substituer ou s'aligner avec la délégation régionale du FASILD (Fonds d'Action et de Soutien pour l'Intégration et la Lutte contre les Discriminations), organisme public qui finance et coordonne les actions des associations qu'elle reconnaît dans le domaine de l'intégration et la lutte contre les discriminations. Finalement, les associations partenaires, voire nouvellement créées évoluent autour de 3 éléments moteurs:

- Claude Bébéar, PDG d'Axa, Président de l'Institut Montaigne, de l'I.M.S, Institut du Mécénat et de la Solidarité, et de l'association Des Entreprises aux couleurs de la France, auteur du rapport du même nom.

- Yazid Sabeg, Président de CS Groupe et de l'association La Clé devenue CREAP, auteur du rapport Les Oubliés de l'égalité des chances, Pour une discrimination positive, et de la «Charte de la diversité».

- Bertrand Collomb, PDG de Lafarge, Président de l'AFEP et chef de projet sur la «Charte de la diversité».

\section{Conclusion : les effets structurants d'une charte}

A partir du postulat selon lequel la gouvernance est un mode de gestion qui se codifie au regard de normes négociées, l'étude de la «Charte de la diversité» nous a permis de nous intéresser à l'idéologie sous-jacente à son apparition, à son processus d'élaboration, et à ses effets structurants. Ainsi, nous avons pu voir que la situation éminemment politique des personnes discriminées en France a été érigée en problématique économique, puisqu'il a été question d'anticiper des pénuries de main d'œuvre, de résorber le chômage dans les zones urbaines, et d'adapter le personnel des entreprises à ses clients. Il est également apparu une sorte de désolidarisation de l'économie et du politique, dans l'évitement du débat politique, dans l'interprétation des causes et conséquences politiques de l'objet au service des enjeux économiques, ainsi qu'au profit des théories de l'insertion par l'économique.

Par ailleurs, l'appropriation de cette thématique par le collectifs d'employeurs a marqué l'abolition de la frontière public / privé, une césure renforcée par l'évitement du débat avec les partenaires sociaux. Le processus de ratification s'est finalement révélé être une pluralité de temps de régulation entre des acteurs d'un groupe social relativement homogène, conduisant à un compromis résultant de l'équilibre de valeurs précaires, à une norme négociée, étrangère aux modes de décision que requiert la démocratie. La diversité tant réclamée n'a donc pas été de mise parmi les principaux protagonistes qui se sont choisis dans un cercle social restreint, unis, comme pour influer davantage et se légitimer auprès des acteurs publics qui deviennent positionnés sur un même niveau horizontal, voir réduits à un rôle d'agents régulateurs.

En définitif, notre analyse de la "Charte de la diversité» nous a permis d'illustrer des attributs de la gouvernance, et de mettre en exergue d'autres caractéristiques de l'objet : 


\section{- La charte produit des normes négociées}

51 Cette charte est le résultat d'un compromis qui promeut des normes de référence et des «bonnes manières de faire» sur la problématique des discriminations dans l'entreprise. Les préceptes d'actions sont formalisés et inscrits dans un objet, ce qui permet d'éviter que l'on interroge à tout instant sa cohérence logique. Il apparaît assez clairement que le choix de formaliser des engagements par le biais d'une charte cristallise un certain sens de l'évolution des rapports de force entre l'entreprise et ses partenaires. En effet, le collectif d'entrepreneurs est parvenu à faire dominer son discours et a également réussi à écarter la présence des organisations syndicales (pour un temps au moins). La charte n'est en finalité que le produit de la relative homogénéité d'un groupe social de référence (les entrepreneurs) et n'a pas résulté d'une mise en entente entre différents acteurs sociaux alors même que les principes d'actions sont susceptibles de s'appliquer à des formes sociales hétérogènes. En effet, il apparaît que cette charte précède l'injonction réglementaire et est une manière pour les entreprises de prouver leur capacité à s'autoréguler.

De plus, il apparaît que la charte représente la résultante d'un équilibre de valeurs précaires qui sont négociées (explicitement ou non) dans le cadre de rapports politiques et sociaux conflictuels et en constantes évolutions. Autrement dit la charte est un objet tributaire de la mobilité des rapports sociaux (l'objet est régulièrement remis en cause ; la charte n'est aussi peut être qu'un effet de mode). Dès lors, nous pourrions formuler l'hypothèse qu'une autre forme de régulation prenne le pas sur celle des chartes. Or de tels changements ne sont pas seulement dus à des valeurs (préférences et intérêts des acteurs qui se modifient), mais c'est parce que les rapports de pouvoir changent ; « $\mathrm{Si}$ l'acteur $\mathrm{A}$ a plus de moyens de contraindre $\mathrm{B}$, les préférences (les valeurs) de A tiendront plus de place dans la régulation $\mathrm{AB}$. Plus généralement, si la régulation est un compromis entre les rationalités des deux groupes, elle pourra se modifier non seulement quand ces rationalités changent, mais quand les pouvoirs respectifs des deux groupes se modifient » [Reynaud, 1997].

53 Par ailleurs, ne disposant d'aucun statut juridique reconnu, cette norme négociée tient lieu de promesse, son « inobservation » n'engendrant aucune conséquence au regard de la loi. La charte est un ensemble de volontés formulées par le collectif qui décide de "se prescrire à soi-même ». Ils entendent remplir des devoirs (pas toujours explicites) au delà de l'autorité légale. Cette charte est donc bien cette promesse de l'entreprise faite à certaines de ses parties prenantes et surtout à l'égard des pouvoirs publics et du réseau associatif.

\section{- La charte est un objet pour l'action}

La charte ne se suffit pas à elle-même, elle n'est qu'un premier pas mobilisateur avant l'action collective. En effet, dans une lettre écrite par Bertrand Collomb et envoyée aux présidents des entreprises de l'AFEP, des entreprises non présentes lors des réunions d'élaboration de la charte sont invitées à rallier l'initiative : «Pourriez-vous nous faire part de votre observation sur ces projets de document (ci-joint), m'indiquer votre soutien (...) et votre position sur la signature par votre entreprise de la "Charte de la diversité»" [extrait lettre d'accompagnement de la charte adressée aux signataires]. 

diversité cherche à se mettre en place. D'ailleurs, lors de ces réunions, les entreprises ont travaillé sur les projets de :

- «Charte de la diversité»

- Cahier des charges pour un label de la diversité

- Note visant à permettre aux entreprises d'établir une évaluation statistique de la diversité.

Ainsi, le collectif d'entrepreneurs cherche à se coordonner pour élaborer des actions communes en matière de lutte contre les discriminations en labellisant des démarches. La charte est donc bien un prétexte pour l'action. Par ailleurs, la lettre de Bertrand Collomb évoque explicitement l'élargissement de la structure du collectif de départ : «Le groupe de travail a privilégié la piste d'un réseau d'entreprises doté d'une structure légère, agissant en " partenariats professionnels » avec des associations existantes ».

lettre est accompagnée d'un document recensant les associations oeuvrant sur ce terrain. La structure du collectif s'élargit et s'ouvre à d'autres partenaires devenus indispensables pour opérationnaliser les engagements figurant dans la charte. La logique de cooptation prévaut encore.

D'autre part, nous avons rencontré Catherine Ferrant, responsable de la diversité et de l'innovation sociale du groupe Total. Elle nous a expliqué que l'entreprise avait été très sollicitée par des associations depuis la signature de la charte. L'entreprise est également sollicitée en interne : les organisations syndicales sont susceptibles de se saisir de cette déclaration d'intention pour interpeller l'entreprise. Un accord sur la diversité est d'ailleurs en cours d'élaboration. Par ailleurs, la raffinerie du groupe Total basée dans le Nord Pas de Calais a été invitée à participer à la conférence sur la diversité de Lille. Pour Catherine Ferrant cette charte "[devait] être déclinée, ça permet de faire des événements régionaux, et qu'on en parle (...) il y aura des articles dans le Voix du Nord (...) et c'est aussi l'occasion d'impliquer des petites entreprises et des PME. Je crois que c'est ça aussi l'idée, il y a un rôle d'entraînement des grandes entreprises vis-à-vis des PME dans le tissu industriel local» (entretien Total).

Outre la caractérisation de l'objet de charte, cette étude de cas nous a donc permis de mieux appréhender la gouvernance. Ses outils et procédés, caractéristiques d'une certaine idéologie se répandent, voire se propagent par l'action. En effet, initiée par l'Institut Montaigne, la «Charte de la diversité» est ensuite travaillée par l'AFEP, signée par des entreprises extérieures à l'AFEP, puis à leur délégations régionales, puis par des PME à l'occasion de cérémonies locales, et enfin donne naissance à d'autres chartes élaborées en interne. Finalement, ce sont bien des normes qui sont diffusées parmi un grand nombre d'entreprises, et avec leur acceptation, la diffusion d'une idéologie capable de peser sur la situation économique, sociale et politique du pays. Dès lors, nous pouvons nous demander si, tout comme la charte est un objet pour l'action, la gouvernance n'est pas une idéologie pour l'action. Enfin, pour en revenir au titre de notre article, « chartes et gouvernance ", nous pensons que la charte est un support privilégié de la gouvernance. Elle est un objet de régulation et de mobilisation, et se prête aisément à la diffusion. Avec le document, sont diffusées des normes et valeurs fondatrices d'une idéologie. C'est cette même idéologie qui justifie la préférence de la " gouvernance » au « gouvernement ». 


\section{BIBLIOGRAPHIE}

Aknin (A.), Froger (G.), Géronimi (V.), Méral (Ph.), Schembri (P.), « Développement durable :

enjeux, regards et perspectives », in Cahier du GEMDEV, N²8, Avril 2002.

Ballet (J.), De Bry (F.), L'entreprise et l'éthique, Paris, Point, 2001.

Ballet (J.), Les entreprises d'insertion, Paris, PUF, 1997.

Bataille (P.), Interview, « Le gouvernement se saisit de la question de la discrimination au travail, in Journal La Tribune, 26/11/2004, p.12.

Bataille (P.), Le racisme au travail, Paris, La Découverte, 1997.

Bébéar (C.), Des entreprises aux couleurs de la France, Rapport au premier Ministre, La

Documentation Française, 2004.

Boltanski (L.), Thévenot (L.), De la justification, les économies de la grandeur, Paris, Gallimard, 1991.

Capron (M.), Quairel-Lanoizelée, Mythes et réalités de l'entreprise responsable, Paris, La Découverte, 2004.

Cassen (B.), « Le piège de la gouvernance », in Le Monde Diplomatique, Juin 2001, p.28.

Castel (R.), Les métamorphoses de la question sociale, Paris, Fayard, 1995.

Chavagneux (C.), «Le grand bazar de la gouvernance », in Alternatives Économiques, Hors série ${ }^{\circ}$ $59,1^{\mathrm{er}}$ trimestre 2004, pp.52-55.

D'Almeida (N.), L'entreprise à responsabilité illimitée, la citoyenneté d'entreprise en questions, RueilMalmaison, Ed. Liaisons, 1999.

D’Almeida (N.), Les promesses de la communication, Paris, PUF, 2001.

De la Broise (P.), Lamarche (T.) (Dir.) et Huët (R.) (Coord.), Responsabilité sociale : vers une nouvelle communication des entreprises?, Lille, Septentrion, Sous presse.

Desbarats (I.), « Codes de conduite et chartes éthiques des entreprises privées. Regard sur une pratique en expansion », in La Semaine Juridique, Edition Générale, Nㅜ9, 26/02/2003, pp.337-343.

Descolonges (M.), Saincy (B.), Les entreprises seront-elles un jour responsables?, Paris, La Dispute, 2004.

Egan (M.), Constructing a European Market, Oxford, Oxford University Press, 2001.

Garner-Moyer (H.), Apparence physique et GRH, entre choix et discrimination, Paris, Cahier du CERGOR, 2004.

Garner-Moyer (H.), Quand l'école est finie ... les premiers pas de la génération 98, Paris, CEREQ, 2004.

Hermet (G.), La gouvernance- Un concept et ses applications, Paris, Karthala, 2005.

Huët (R.), « Les chartes sociales dans les organisations ", in De la Broise (P.), Lamarche (T.) (Dir.) et Huët (R.) (Coord.), Responsabilité sociale : vers une nouvelle communication des entreprises ?, Lille, Septentrion, Sous presse.

Lascoumes (P.), Le Galès (P.) (Dir.), Gouverner par les instruments, Paris, Sciences Po, 2004. 
Le Galès (P.), « Du gouvernement des villes à la gouvernance urbaine », in Revue Française des sciences politiques, $\mathrm{N}^{\circ} 155$, Mars 1998, p.64.

Lefort (C.), L'invention démocratique : les limites de la domination totalitaire, Paris, Fayard, 1981.

Loneux (C.), « Les chartes éthiques comme outils de communication institutionnelle », in Delcambre (P.) (Dir.), Communications organisationnelles : objets, pratiques, dispositifs, Rennes, PUR, 2000, pp. 122-148.

Loriol (M.) (Dir.), Qu'est ce que l'insertion? Entre pratiques institutionnelles et représentations sociales, Paris, L'Harmattan, 1999.

Muller (P.), Les politiques publiques, Paris, PUF, 2003.

Muller (P.), Surel (Y.), L'analyse des politiques publiques, Paris, Montchrestien, 1998.

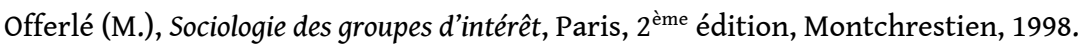

Paugam (S.), (Dir.), L'exclusion, l'état des savoirs, Paris, La Découverte, 1996.

Perez (R.), La gouvernance de l'entreprise, Paris, La Découverte, 2003.

Reynaud (J-D), Les règles du jeu, l'action collective et la régulation sociale, Paris, Armand Colin, 1997.

Sabeg (Y.), Méhaignerie (L.), Les oubliés de l'égalité des chances, Paris, Institut Montaigne, 2004.

Zoonekynd (S.), «Contribution à l'analyse d'une forme singulière d'Entrepreneuriat : le cas des entreprises d'insertion », Revue de l'Entrepreneuriat, Vol.3, n²1, 2004, pp.33-46.

\section{NOTES}

1. Bébéar 2004, Sabeg et Méhaignerie 2004.

2. Nombre d'auteurs ont travaillé sur ce concept de gouvernance et notamment sur son application dans le développement durable, notamment l'article de Aknin, Froger, Géronimi, Méral, et Schembri (2002). Dans notre article, la définition que nous retenons vise à insister sur les transformations structurelles de l'action publique. Cette dernière se caractérise de plus en plus «par du bricolage, de l'enchevêtrement de réseaux, de l'aléatoire, une multiplication d'acteurs, des finalités multiples, de l'hétérogénéité, de la transversalité des problèmes, des changements d'échelles des territoires de référence (...) L'État semble perdre son monopole, il est moins le centre des processus politiques, de régulation des conflits. » [Lascoumes, Le Galès, 2004, p.23]. Ainsi, si les chartes peuvent constituer une forme de gouvernement du social, notre approche est centrée sur le rôle joué par les acteurs privés dans les régulations sociales dans la mesure où ils tendent à se conduire comme des gouvernements [Egan, 2001, p.6].

3. Guy Hermet est Directeur de Recherche émérite à la Fondation nationale des sciences politiques (CERI)

4. L'institut Montaigne est une association d'inspiration libérale, composée pour la majorité de chefs d'entreprises, de hauts fonctionnaires, d'universitaires et de représentants de la société civile. Présidé par Claude Bébéar, l'Institut se présente comme « un laboratoire d'idées » jouant un « rôle d'acteur du débat démocratique ", notamment à travers l'élaboration de propositions et de recommandations sur des enjeux de société.

5. Il s'agit d'une association dont le président est Bertrand Collomb (PDG du groupe Lafarge). Elle réunit 90 grandes entreprises. Ce lieu permet la rencontre des entreprises qui peuvent alors échanger sur leurs pratiques respectives et assister à des conférences organisées par l'association. Elle ne s'occupe pas spécifiquement de la question des discriminations. Il s'agit d'un 
« club d'entreprises » qui cherchent à unifier les revendications portées par les entrepreneurs notamment sur certaines questions sociales.

6. Cf. Bibliographie. Ces auteurs ont travaillé sur la question de la Responsabilité sociale des entreprises.

7. La multiplication des fondations d'entreprises en est un signe. Nicole D'Almeida précise que le principe de fondation consiste à affecter tout ou partie d'une fortune à une œuvre désintéressée, utile au bien commun. Le législateur les a d'ailleurs distinguées des associations de types loi de 1901 en redéfinissant leur statut (personne morale à but non lucratif) leur assignant une mission d'intérêt général (qui n'est pas forcément reconnue d'utilité publique), une durée minimale (5 ans) et une dotation initiale.

8. Cela signifie que la charte pourrait disposer d'un régime juridique connu, pour autant, son contenu général rend son application concrète invérifiable pour le juge.

9. Cette expression désigne avant tout la "scène politique " et les jeux de pouvoir qui s'y rapportent. La participation de certains acteurs sociaux à la signature du document participe à objectiver une problématique sociale car elle témoigne dans une certaine mesure de leur prise de conscience que tel ou tel phénomène intéresse l'avenir de la collectivité et qu'ils sont déterminés à le canaliser, le corriger et lui conférer une certaine organisation.

10. Dès lors, on peut s'interroger sur les raisons de ce refus de donner à ces signatures une valeur juridique alors que chacun des acteurs fait le choix de s'engager dans une direction donnée.

11. Dans les perspectives ouvertes par Offerlé [1998, p.22], nous pourrions dire que l'action de ces chefs d'entreprises ne se limitent pas à mettre en forme et en scène des visions du monde et des revendications, mais qu'ils délivrent à leurs adhérents ou à leurs représentés (dans ce cas, il s'agit plutôt d'adhérents), sans passer par le circuit long de la politique, des prestations concrètes et dotées de réalité (introduction de nouveaux outils de gestion, pression pour modifier certaines dispositions de la loi sur le CNIL, etc.) - Cf. partie 3.

12. C'est donc en qualité d'entrepreneur que Yazid Sabeg intervient au cours des Assises de la politique de la ville. Il affirmera que cette politique ne cessera d'être un échec tant que l'on continuera à occulter les processus de discrimination dans les politiques de renouvellement urbain et d'insertion des populations en difficulté.

13. Orienté car comme le montrent Muller et Surel [1998, Ibid.] «Il n'existe pas une univocité dans la recherche des causes, mais une quête de sens menée par les acteurs. Ces derniers mobilisent et sélectionnent tour à tour les différent registres d'intelligibilité, afin de conférer une signification et/ou donner une explication à un réel chaotique (...) Cette recherche des causes implique un travail cognitif et normatif de sélection des données pertinentes à partir d'une simplification plus ou moins grande des composantes du phénomène considéré, opération ellemême déterminée par les grilles de lecture particulières aux différents acteurs [p.59]. Autrement dit, cela signifie que ces rapports, mais également la charte, véhiculent une conception particulière $\mathrm{du}$ problème des discriminations. Cette conception s'inscrit dans une certaine cohérence avec leurs intérêts, leurs valeurs, et leurs visions du monde. Cela s'est notamment traduit par la production de rapports, d'une charte, mais également par l'introduction de nouveaux termes pour qualifier le fait social (tels que les termes de "minorités visibles", de « diversité » ou « d'action positive à la française » qui ne sont pas axiologiquement neutres). En tout état de cause, l'ensemble de cette production discursive constitue une formulation intelligible de l'implication des entreprises et est donc une manière de «labelliser le problème dans un sens particulier ». Nous reviendrons sur ce point plus bas.

14. Ces rapports ne sont pas exclusivement centrés sur la problématique des discriminations dans le monde économique. D'autres domaines sont abordés tels que la politique de la ville, l'éducation, etc.

15. Les auteurs de la «Charte de la diversité» cherchent à assouplir la loi sur le CNIL. À titre d'exemple, Laurence Méhaignerie (Chargé de recherche, Institut Montaigne, rédactrice de la 
charte) insiste sur le fait qu'il «serait difficile de vouloir transformer la réalité sociale de l'entreprise sans connaître cette réalité (...) l'instrument de mesure permet de disposer d'une photographie statistique et anonyme de la sociologie de l'entreprise. L'entreprise connaît ainsi, à un moment donné, le taux de minorités visibles qu'elle compte dans son effectif. Elle croisera cette information avec des données sur les niveaux hiérarchiques où ces minorités sont les plus présentes. Cette étude a tout intérêt à être répétée dans le temps, afin de suivre les progrès réalisés " [Laurence Méhaignerie, Déjeuner débat du 28/04/2005, Institut du Mécénat et de la Solidarité, Gestion de la diversité dans l'entreprise : des outils statistiques pour mesurer la diversité des origines. Quels obstacles aujourd'hui et quelles solutions pour demain?]

16. Théories selon lesquelles l'insertion de publics en difficulté est rendue possible par l'exercice d'une action professionnelle dans le cadre d'une relation contractuelle de travail. L'idée sousjacente consiste à « recomposer un espace de socialisation professionnelle pour des populations privées de travail ou qui n'y ont jamais accédé (...) l'autonomie de l'individu étant recherchée par sa mise en situation de travail et d'interaction socio-professionnelle dans des conditions réelles [Ballet, 1997, pp.58-59]. Si ces théories sont essentiellement rapportées au rôle des « entreprises d'insertion" (cf, Zoonekynd, 2004, Loriol, 1999, etc.), les analyses sur l'égalité des chances procèdent du même ordre. Notre position consiste à nuancer le principe selon lequel la seule acquisition des revenus et l'évolution dans des relations de travail résoudraient les difficultés d'intégration dans la mesure où ne sont pas pris en compte les processus de ségrégation sociale [voir sur ce point notamment Castel (1995) et Paugam (1996)].

17. Extrait de l'édito du n ${ }^{\circ} 8$ de La Lettre de D.M.A (D'un Monde à l'Autre), septembre 2004, intitulé «L'égalité et l'emploi : des principes et des pratiques ».

18. Le groupe de travail était composé de Laurence Méhaignerie (animatrice), et d'une vingtaine d'entreprise membres de l'AFEP. Aucun acteur extérieur à l'association n'a participé au processus d'élaboration de la charte. Selon l'animatrice, à chaque réunion, il y avait approximativement 20 chefs d'entreprise.

19. L'Association Alliances est composée d'entrepreneurs et vise à sensibiliser les entreprises de la région du Nord Pas de Calais à leurs responsabilités sociales (www.alliances-asso.org)

\section{RÉSUMÉS}

Notre travail consiste à mettre à l'épreuve du terrain le concept de gouvernance, entendu comme une forme d'intervention des entreprises dans l'action publique. À travers la problématique des discriminations (fondées sur l'origine ethnique) dans le monde du travail, nous montrerons comment une question sociale a été saisie par des acteurs privés auteurs de différentes initiatives telles que la production de rapports dédiés à ce sujet (Bébéar 2004, Sabeg et Méhaignerie 2004...) et à travers la récente signature de la "Charte de la diversité» par une quarantaine d'entreprises françaises en octobre 2004. Ces différentes initiatives (production de rapport, d'une charte, etc.) révèlent la tentation des acteurs privés à ériger un problème public, à influer sur les relations étatiques, à légitimer et à supporter leurs propres explications du réel, des différentes causalités ainsi que des solutions qui s'y rapportent. L'appropriation de cette question des discriminations par les acteurs privés témoigne de leur participation croissante dans les décisions politiques. Elle illustre donc un mode de gestion qui se codifie au regard de normes négociées, et caractérise en partie les outils et procédés de la gouvernance. Ainsi, à partir du postulat selon lequel la gouvernance est un mode de gestion qui se codifie au regard de normes négociées, nous avons 
mené une étude sur la "Charte de la diversité » dans les entreprises rendue publique en octobre 2004. Ce travail permet également de questionner la participation et le rôle des entreprises dans le développement de politiques qui ont trait à la justice sociale et à l'équité. Plus que de " développement durable», on évoque le terme de "responsabilité sociale des entreprises" pour signifier les devoirs qui « s'imposent » à ces organisations économiques.

The objective of our paper consists in testing on the ground the concept of governance, which should be understood as a form of intervention by companies in public action. Using the social problem of "racial" discrimination in the workplace, we will show how this has been taken up by private players in initiatives such as the production of reports on the subject (Bébéar 2004, Sabeg and Méhaignerie 2004...) and in the recent signing of the "Charter of diversity" by forty french firms. In fact, these various initiatives (reports, charters...), show how private players are tempted to build on a public problem, to influence relations with the State, to legitimise and support their own explanations of what is real, their own ways of defining the causes, and their solutions. The fact that the question of discrimination has been appropriated by private players is a testimony to their increasing participation in political decision-making, and thus illustrates a way of working which is codified from the point of view of a negotiated standard, and partly characterised by the tools and procedures of governance. Thus, starting from the premise that governance is a way of working codified from the point of view of a negotiated standard, we have studied the Charter of Diversity in business which was published in October 2004.

INDEX

Mots-clés : gouvernance, responsabilité sociale des entreprises, chartes et codes de conduite, charte de la diversité, action publique, discriminations, communication

\section{AUTEURS}

\section{ROMAIN HUËT}

Doctorant en Sciences de l'Information et de la Communication, GERIICO (Université Lille 3) et Centre de Recherche en Éthique Économique de Lille (Université Catholique de Lille)

\section{MORGANE CANTRELLE}

Master 2 « Communication et développement des territoires », Université Lille 3 